\title{
A capsulotomia descontínua durante a facoemulsificação
}

\section{Discontinuous capsulotomy during phacoemulsification}

Virgilio Centurion'; Augusto Cézar Lacava'; Juan Carlos Caballero

\section{RESUMO}

Objetivo: Descrever a prevalência, localização e causas da capsulotomia descontínua durante a cirurgia de catarata por facoemulsificação. Métodos: Estudo retrospectivo, consecutivo, não comparativo de 971 cirurgias de catarata com capsulotomia descontínua no per-cirúrgico entre janeiro de 2009 a junho de 2010. Resultados: Observou-se uma prevalência de $0,02 \%$ e a descontinuação da cápsula ocorreu em $100 \%$ das vezes durante o tratamento do núcleo. Em 58.33\% dos olhos o implante foi realizado no saco capsular e em 41.67\% no sulco ciliar. A AV $\geq 20 / 30$ em 100\% dos olhos. Conclusão: apesar de ser uma intercorrência pouco frequente, pode comprometer o resultado final.

Descritores: Lentes intraoculares; Extração de catarata/métodos; Facoemulsificação/métodos; Capsulorrexe/ métodos

\section{AbStract}

Objective: To describe the prevalence, location and causes of discontinuous capsulotomy during phacoemulsification cataract surgery. Methods: Retrospective, consecutive, non-comparative study of 971 cataract surgeries - with discontinuous capsulotomy during surgery - performed from January 2009 to June 2010. Results: it was observed a prevalence of $0.02 \%$ and capsule discontinuation occurring $100 \%$ of the time during the treatment of the nucleus. In $58.33 \%$ of the eyes the implant was done in the capsular bag and in $41.67 \%$ in the ciliary sulcus. VA $\geq 20 / 30$ in $100 \%$ of the eyes. Conclusion: despite the fact that it is a not too frequent event, it may compromise the final result. methods

Keywords: Lenses, intraocular; Cataract extraction/methods; Phacoemulsification/methods; Capsulorhexis/

\footnotetext{
${ }^{1}$ Oftalmologistas do Instituto de Moléstias Oculares - IMO - São Paulo (SP), Brasil

Trabalho realizado no Instituto de Moléstias Oculares - IMO - São Paulo (SP), Brasil

Os autores declaram inexistir conflitos de interesse
} 


\section{INTRODUÇÃO}

A facoemulsificação é uma técnica considerada etapa-dependente, ou seja, todas as fases devem ser realizadas sem complicações, pois a imperfeita realização de uma delas pode comprometer o restante da técnica cirúrgica ou o resultado final. Assim, a capsulorrexe deve ser realizada seguindo o objetivo de ser circular, contínua, central e de uma circunferência de aproximadamente 5,0 a $5,5 \mathrm{~mm}$.

Quando tem a sua estrutura comprometida podemos ter complicações intraoperatórias como ruptura da cápsula anterior e/ou posterior, perda vítrea ${ }^{(1)}$, fragmentos cristalinianos no vítreo, ausência de suporte para LIO e deslocamento da LIO no pós-operatório.

A capsulorrexe descontínua pode ocorrer durante a confecção da capsulorrexe ou em qualquer estágio da facoemulsificação.

Objetivo deste trabalho foi descrever a prevalência, a localização mais frequente, a possível causa desta complicação e suas consequências.

\section{MÉTOdos}

Trata-se de um estudo retrospectivo, consecutivo e não comparativo de olhos submetidos à facoemulsificação por catarata e com diagnóstico de lesão da cápsula anterior do saco capsular e que receberam implante de LIO, com injetor, no saco capsular ou no sulcus ciliaris, no período entre janeiro de 2009 a junho de 2010

Avaliamos as causas, as complicações per e pósoperatórias, acuidade visual final e estabilidade da LIO no local implantado.

Todas as cirurgias foram realizadas por um único cirurgião (V.C.), sob anestesia peribulbar, incisão corneana de $2,75 \mathrm{~mm}$ a hora 10 , facoemulsificação com o equipamento INFINITI Vision System - Alcon e implante de LIO com injetor no saco capsular ou no sulcus ciliaris.

\section{Resultados}

Entre janeiro de 2009 a junho de 2010 foram analisados prontuários de 971 cirurgias de catarata. Desse total 12 olhos apresentaram capsulotomia descontínua, com prevalência de $0,02 \%$ (Tabela 1 ).

Em relação à etiologia, 100\% dos olhos (12) apresentaram capsulotomia descontínua durante o tratamento do núcleo pela facoemulsificação e nenhum olho du- rante a confecção da capsulotomia. O local da lesão da cápsula anterior podemos observar na Tabela 2 .

Ao analisarmos o local do implante da LIO, em 7 olhos $(18,33 \%)$ a LIO foi implantada no saco capsular e em 5 olhos (41,67\%) no sulcus ciliaris (Tabela 3). O modelo de LIO implantada se observa na Tabela 4.

As complicações operatórias observadas foram: 1 olho com ruptura de cápsula posterior sem perda vítrea e 1 olho com posteriorização da capsulotomia sem perda vítrea.

A acuidade visual pós-operatória foi de $\geq 20 / 30$ em $100 \%$ dos olhos com capsulotomia descontínua. O seguimento variou de 3 meses a 1 ano e 6 meses.

\section{DISCUSSÃO}

A confecção da capsulorrexe é um passo importante na facoemulsificação. O treino em olhos de porco, nem sempre simula as condições humanas, e já existe um simulador cirúrgico para treinamento da confecção da capsulorrexe $\mathrm{e}^{(2)}$.

A capsulorrexe curvilínea contínua (CCC) é mais resistente à formação de rasgos radiais da cápsula ${ }^{(3,4)}$. Ela permite ao cirurgião acesso ao material cristaliniano a ser retirado e depois ao implante da LIO. Uma intacta cápsula posterior serve de barreira entre os segmentos anterior e posterior do globo ocular. Não deve ser muito pequena, pois pode predispor à síndrome da contração capsular e quando muito ampla pode permitir a captura da $\mathrm{LIO}^{(5,6)}$.

Marques $^{(1)}$ observou 21 casos de descontinuação da cápsula e/ou ruptura da cápsula anterior em 2646 cirurgias, ou seja uma prevalência de $0,79 \%$. Esta alteração foi observada em 1 olho durante a injeção do viscoelástico, em 13 olhos durante a confecção da capsulorrexe, em 2 olhos durante a hidrodissecção, em 3 olhos durante o tratamento do núcleo, em 1 olho durante a irrigação/aspiração e em 1 olho durante a implantação de gancho de íris. Em 14 cirurgias a descontinuação capsular se estendeu até a zônula, em 10 se estendeu até o equador e cápsula posterior. A vitrectomia anterior foi necessária em 4 cirurgias. Foi implantada LIO peça única de acrílico em 18 cirurgias e em 3 olhos a LIO de três peças de acrílico.

Em nosso estudo, observamos a cápsula descontínua em 12 olhos num total de 971 cirurgias ou uma prevalência de $0,02 \%$ e todas as lesões da cápsula anterior ocorreram durante a etapa de facoemulsificação (Tabelas 5 e 6). O local da lesão da cápsula anterior foi dividido igualmente nos quatro quadrantes. 
Tabela 1

Total de cirurgias e prevalência da capsulotomia descontínua

- período: de janeiro de 2009 a junho de 2010 (16 meses)

- total de cirurgias: 971

- total de capsulotomias descontínuas: 12 olhos

- prevalência: $0,02 \%$
Tabela 3

Local do implante

\begin{tabular}{lcc}
\hline Local do implante & Olhos & \% \\
\hline Saco capsular & 7 & 58,33 \\
Sulcus ciliaris & 5 & 41,67 \\
Total & 12 & 100,00 \\
\hline
\end{tabular}

Tabela 2

Local da lesão da capsula anterior

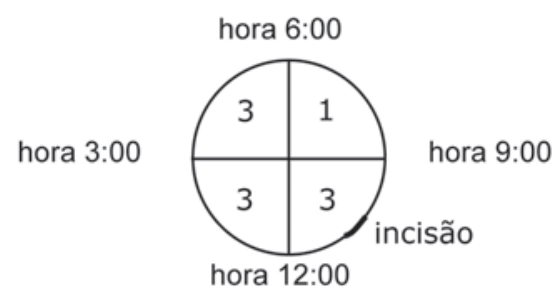

Posição do cirurgião

Tabela 4

Material da LIO implantada

\begin{tabular}{lcc}
\hline LIO & Olhos & $\%$ \\
\hline LIO silicone 3 peças & 7 & 58,33 \\
LIO acrílica hidrofóbica 3 peças & 2 & 16,67 \\
LIO acrílica hidrofóbica peça única & 3 & 25,00 \\
Total & 12 & 100,00 \\
\hline
\end{tabular}

Tabela 5

Prevalência da capsulotomia descontínua de dois autores

\begin{tabular}{lccccc}
\hline Autores & Olhos & $\begin{array}{c}\text { Capsulotomia } \\
\text { descontínua }\end{array}$ & Prevalência (\%) & Seguimento & Ano \\
\hline Marques & 24616 & 21 & 0,79 & 5 anos & 2006 \\
Centurion & 971 & 12 & 0,02 & 1 a e 6 meses & 2010 \\
\hline
\end{tabular}

Tabela 6

Etapa da cirurgia em que foi provocada a capsulotomia descontínua

\begin{tabular}{lcccccc}
\hline Autores & $\begin{array}{c}\text { Injeção } \\
\text { viscoelástico }\end{array}$ & Capsulotomia & Hidro & Faco & I/A & Outros \\
\hline Marques & 1 & 13 & 2 & 3 & 1 & 1 \\
Centurion & - & - & - & 12 & - & - \\
\hline
\end{tabular}


O implante da LIO foi no saco capsular em 7 olhos $(58,33 \%)$ e em 5 no sulcus ciliaris (41,67\%). Em 1 olho houve ruptura da cápsula posterior sem perda vítrea. $\mathrm{Na}$ maioria das cirurgias $(75 \%)$ houve preferência pelo implante de LIO monofocal de 3 peças. É importante notar que a $A V \geq 20 / 30$ aconteceu em $100 \%$ dos casos e não houve complicações no pós-operatório.

Assia $^{(3)}$ mostrou que $95 \%$ dos rasgos da cápsula se estendem até a periferia, não ultrapassando o equador. Dois fatores seriam responsáveis pela limitação da extensão da descontinuação da capsulotomia: as características anatômicas da zônula e a direção da força exercida.

A disposição e estrutura das zônulas funcionam como uma verdadeira barreira à progressão do rasgo da cápsula, pois cada zônula cobre grande parte da cápsula e qualquer parte da periferia da cápsula é amparada por várias zônulas ${ }^{(3)}$.

Durante a etapa do tratamento do núcleo pela facoemulsificação, o uso de alto vácuo, aspiração alta e pressão de irrigação elevada, podem provocar injuria $\operatorname{zonular}^{(7)}$.

Acreditamos que a diferença na prevalência da capsulotomia anterior descontínua entre os dois autores se deva à diferença de períodos comparados e critérios das causas.

Davison $^{(8)}$ sugere colocar as alças da LIO perpendicular à área de ruptura.

Isto conduz a um protocolo de segurança em relação à LIO que o cirurgião deve ter de reserva no centro operatório. Em tempos de lentes Premium (esféricas, tóricas, multifocais, acomodativas) a possibilidade de comprometimento da capsulotomia anterior e/ou outro tipo de complicação nos obriga a ter lentes de reserva, na dioptria adequada para implante no saco ou sulcus ciliaris.
Em conclusão, trata-se de uma intercorrência pouco frequente, que não compromete o resultado da acuidade visual final, porém se este tipo de complicação se apresentar durante uma cirurgia com indicação de implante de lentes Premium a ausência da integridade do saco capsular pode comprometer não somente o implante previamente programado como também o resultado final.

\section{REFERÊNCIAS}

1. Marques FF, Marques DM, Osher RH, Osher JM. Fate of anterior capsule tears during cataract surgey. J Cataract Refract Surg. 2006;32(10):1638-42.

2. Privett B, Greenlee E, Rogers G, Oetting TA. Construct validity of a surgical simulator as a valid model for capsulorhexis training. J Cataract Refract Surg. 2010;36(11):1835-8.

3. Assia EI, Apple DJ, Tsai JC, Morgan RC. Mechanism of radial tear formation and extension after anterior capsulectomy. Ophthalmology. 1991;98(4):432-7.

4. Werner L, Jia G, Sussman G, Maddula S, Ness P, Davis D, et al. Mechanized model to assess capsulorhexis resistance to tearing. J Cataract Refract Surg. 2010;36(11):1954-9.

5. Chang DF. Spotlight on cataract surgery: what would you do? EyeNet. 2010. p.47-61. [cited 2011 Jun23]. Available from: http://www.eyecareamerica.org/isrs/upload/ Spotlight_Cataract_Surgery.pdf

6. Stark WJ, Streeten B. The anterior capsulotomy of extracapsular cataract extraction. Ophthalmic Surg. 1984;15(11):911-7.

7. Coelho RP, Zanatto MC, Paula JS, Romão E. Spontaneous late in-the-bag intraocular lens dislocation after can-opener capsulotomy: case report. Arq Bras Oftalmol. 2005;68(6):864-6.

8. Davison JA. Analysis of capsular bag defects and intraocular lens positions for consistent centration. J Cataract Refract Surg. 1986;12(2):124-9.
Endereço para correspondência:
Virgilio Centurion
Av. Ibirapuera, $n^{0} 624$ - Ibirapuera
CEP 04028-000 - São Paulo (SP), Brasil
E-mail: centurion@imo.com.br 